\title{
RESONANCE CALCULATION BASED ON THE GLOBAL-LOCAL METHOD FOR THE COMPLEX GEOMETRY PROBLEMS
}

\author{
Lu Cao, Zhouyu Liu*, Qingming He, and Liangzhi Cao \\ School of Nuclear Science and Technology, Xi'an Jiaotong University \\ 28 West Xianning Road, Xi'an, Shaanxi 710049, China \\ *zhouyuliu@xjtu.edu.cn
}

\begin{abstract}
With the development of nuclear reactor, new complicated designs introduce some challenges for the resonance method in the NECP-X code, which is called as the Global-Local method. There are two limitations of the present NECP-X. One is the geometry modeling limitation, and the other one is that the Global-Local resonance method can only deal with the cylindrical fuel rods in the current version. Therefore, some fuels like plate, annular fuels cannot be calculated in the NECP-X code because of these limitations. To overcome above issues, the constructive solid geometry (CSG) method is developed to model the complex problem, and the capability of constructing and solving the equivalent 1D plate and 1D annual fuel pin based on the framework of Global-Local method, is developed. A set of tests are calculated, including multi-annular fuel problems and plate fuel problems. The results show good accuracy of the new developed resonance self-shielding method.
\end{abstract}

KEYWORDS: NECP-X, Global-Local, complex geometry, CSG

\section{INTRODUCTION}

The NECP-X [1] code is a high-fidelity deterministic neutronics code developed by the NECP team (Nuclear Engineering Computational Physics Lab.) of Xi'an Jiaotong University. It uses the Global-Local self-shielding calculation scheme [2] to treat resonance, solves the 3D whole core transport problem with the 2D/1D coupling method [3-5], and accelerates the transport calculation by a new free-matrix CMFD acceleration method.

For the self-shielding calculation, the Global-Local method in the current NECP-X code can handle the cylinder geometry. For typical PWR problems, this method shows very high accuracy and good efficiency. However, it encounters difficulties when calculating reactors using the multi-annular or plate fuels. To address this problem, at first, the improved CSG (Constructive Solid Geometry) method is applied in the NECP-X code [6]. Then functionality extension of the Global-Local self-shielding calculation scheme is developed for the multi-annular and plate fuel problems.

The rest of the paper is organized as follows: Section 2 introduces the improvements for the Global-Local calculation scheme. Section 3 demonstrates the accuracy of the scheme by numerical results. The conclusions and summations are given in Section 4. 


\section{GLOBAL-LOCAL METHOD FOR THE COMPLEX GEOMETRY PROBLEMS}

\subsection{Geometry modeling by the improved CSG method}

The CSG method is adopted to build the complicated solid model in Euclidean by some Boolean operations of the basic primitive solid set typically includes: Rectangular-prism (Block), Cylinder, Cone and Sphere and so on. In this work, there are some improvements based on the traditional CSG method for the transport calculations in the deterministic method.

\subsubsection{The auto-mesh functionality}

The NECP-X code adopts 2D/1D transport method, where the MOC (Method of Characteristics) is adopted for 2D radial planar calculations. Since MOC calculation requires large number of flat source regions, manually defining flat source regions one by one would be very time-consuming and error-prone, thus the automatic mesh functionality is developed in NECP-X based on the traditional CSG modeling.

Fig.1 shows a projection of a typical PWR cell on the X-Y plane. For the flat source region 1 shown in Fig. 1 , it can be uniquely determined by plane $\mathrm{AO}$ and $\mathrm{BO}$ which are perpendicular to XOY plane and cylinder $\mathrm{R} 1$ which is parallel to z-axis. Let the angle between $\mathrm{AO}$ and $\mathrm{OX}$ be $\alpha$, the angle between $\mathrm{BO}$ and $\mathrm{OX}$ be $\beta$. Then add $1.0 \times \cos ((\alpha+\beta))$ and $1.0 \times \sin ((\alpha+\beta))$ to the $\mathrm{X}$ and $\mathrm{Y}$ coordinates of point $\mathrm{O}$ (the center point) to get point $\mathrm{P}$. Subtract the coordinates of $\mathrm{P}$ in $\mathrm{AO}, \mathrm{BO}$ surface equation and positive or negative half-space information for building flat source region 1 can be obtained. Through this procedure, the flat source regions can be generated automatically by supporting information likes angles, radius, region center point, etc.

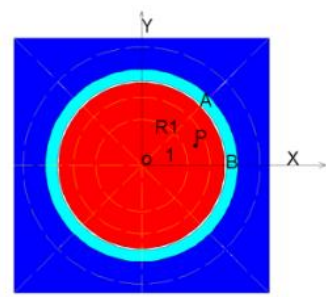

Figure 1. Material regions and flat source regions of a typical PWR pin cell

\subsubsection{The volume calculation}

The random points sampling method and the method based on characteristic ray information are used for the volume calculation in NECP-X. The random point sampling method uses stochastic points to get the volume based on the probability theory. The method based on the characteristic ray information firstly needs the characteristic segments for all flat source regions, then the volume is calculated by the method of Newton interpolation polynomials [7].

\subsection{The Global calculations}

The Dancoff correction factor of each fuel pin is obtained by NCM (Neutron Current Method) [8]. The Dancoff correction factor of the target fuel pin is calculated as:

$$
D=\frac{\phi_{S, 0}-\phi_{S, 1}}{\phi_{S, 0}}
$$

Where $D$ is the Dancoff correction factor of fuel; $\phi_{S, 0}$ is the flux of fuel in the isolated system; $\phi_{S, 1}$ is the flux of fuel in the target problem system. 
It should be noted that the fuel pin here includes the cylindrical fuel, the multi-annular fuel and the plate fuel. Therefore, the isolated system for the cylindrical and multi-annular fuel pin is a 1D cylindrical problem, and 1D plate problem for the plate fuel case. Firstly, the $\phi_{S, 0}$ and $\phi_{S, 1}$ are obtained by solving the Equation (2) at the isolated system and the target problem system, respectively.

$$
\Omega \nabla \phi(r, \Omega)+\Sigma_{t}(r) \phi(r, \Omega)=S(r)
$$

Where $\phi(r, \Omega)$ is the angular flux; $\Sigma_{t}(r)$ is the total macroscopic cross section; $S(r)$ is the fixed neutron source.

There are some assumptions used in the calculation of $\phi_{S, 0}$ and $\phi_{S, 1}$. Normally, for cylindrical and plate fuel pin, the fuel regions are treated as black which means its total cross section is very large, and 1.0e5 $\mathrm{cm}-1$ is used in the NECP-X code, and the scattering cross section is assumed to be $0.0 \mathrm{~cm}-1$. The fixed neutron source is set to be zero in the fuel regions. For other regions, the total cross section is set to be the potential scattering cross section and the scattering cross section is set to be zero. The fixed neutron source is set to be equal to the value of the potential scattering cross section.

However, for the multi-annular fuel problems, there are some non-fuel regions between the fuel regions in a fuel pin. It is assumed in this work that the regions between fuel regions are treated as fuels when calculating the Dancoff correction factor. Therefore, the calculation of the Dancoff correction factors of the multi-annular fuel problems can be transferred to the cylindrical fuel problems as shown in the Fig. 1. And the same Dancoff correction factor is used in the fuels inside a pin.

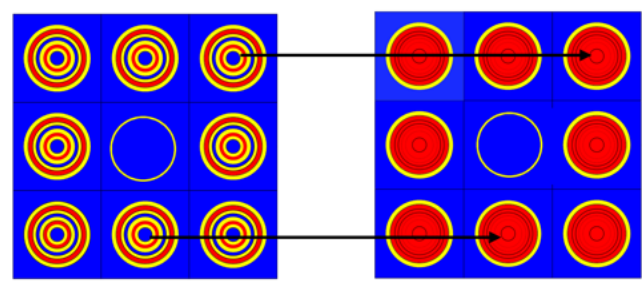

\section{Target Problem \\ Assume Problem \\ Figure 1. The conversion process for multi-annular fuel problem}

\subsection{The coupling scheme}

A binary search of the outer boundary is performed to find the 1D cylindrical problem or the 1D plate problem, whose Dancoff correction factor is equal to that of the target fuel pin obtained in the global calculations. When searching the equivalent 1D cylindrical problem, the Dancoff factor of the 1D cylindrical or the 1D plate pin cell can be expressed as

$$
D=f(L)=\frac{P_{e, i}-P_{m s}(L)}{\Sigma_{t s} l P_{m s}(L)+P_{e, i}-P_{m s}(L)}
$$

Where:

$P_{e, i}$ is the escape probability from the isolated fuel pin;

$P_{m s}$ is the collision probability from the fuel region to the other region;

$L$ has different meaning for different problem. It can be the outer radius of the moderator of the 1D cylindrical pin cell for cylindrical or multi-annular fuel pin cell. And for the 1D plate pin cell it is the pitch of the outer moderator; 
$\Sigma_{t s}$ is the total cross section of the fuel region for the plate and cylindrical fuel problem, and it is the total cross section of the regions with the black-body assumption in multi-annular fuel problems;

$l$ is the average chord length of the fuel pin.

Since the Equation (2) and (3) should be of the same value, and Dancoff correction factor defined by Equation (2) is already obtained in the chapter 2.2, $L$ defined in Equation (3) can be obtained by binary search.

The detail processes of different kinds of geometry are shown at Fig. 2 and Fig. 3.

With the $L$ searched by the above process, an equivalent-1D-pin problem is established. The new selfshielded cross sections of the target fuel in the global system could be calculated by solving this new established 1D pin problem.

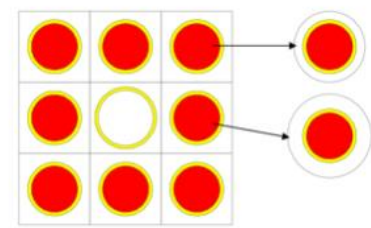

Figure 2. The Equivalence between a fuel pin cell in lattice and a 1D cylindrical fuel pin cell

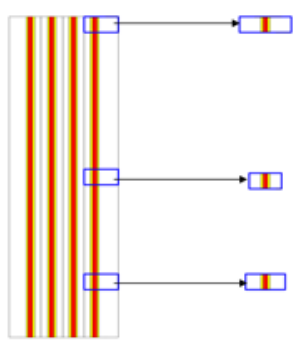

Figure 3. The Equivalence between a fuel pin cell in lattice and a 1D plate fuel pin cell

\subsection{The Local calculations}

The pseudo-resonant-nuclide subgroup method (PRNSM), which is introduced in detail in the previous work in our team [9], is used to solve the equivalent $1 \mathrm{D}$ pin cell problem. The method can be mainly divided into the following steps:

- Process the cross section for the pseudo-resonant nuclide by giving a set of concentrations of resonant isotopes, which are mixed with the pseudo-resonant nuclide;

- The subgroup parameters of the pseudo-resonant nuclides are obtained by the least square fitting method and the subgroup cross sections of each resonant nuclide are obtained;

- The effective self-shielding cross sections of each resonant nuclide are obtained by solving the subgroup fixed source problem;

- SPH correction is adopted to ensure the conservation of reaction rate in the transport calculation.

\section{NUMERICAL RESULTS AND ANALYSIS}

The reference solution in this paper is calculated by the MC (Monte Carlo) code McCARD [10]. The ENDF/B-VII.0 library is used both in these two codes. The WLUP-69 energy group structure [11] is 
adopted in NEPC-X, and it should be noted that the resonance energy group of the NECP-X code is extended to $0.625 \mathrm{eV} \sim 24780 \mathrm{eV}$, which is located from the 13 th group to the 45 th group of the energy group.

\subsection{A multi-annular fuel pin cell problem}

The multi-annular fuel problem is a $\mathrm{UO}_{2}$ fuel plate with $3.1 \mathrm{wt} \% \mathrm{U}-235$. As shown in Fig. 4, Natural zirconium and the light water are used for the materials of the clad and the moderator, respectively. The temperatures of all regions are $600.0 \mathrm{~K}$ and reflective boundary condition is considered in the calculation. The radii from the inner ring to the outer ring are $0.1,0.15,0.2,0.25,0.3,0.35,0.4,0.475 \mathrm{~cm}$.

Fig. 6 shows the relative difference of group-wised microscopic absorption cross-section of ${ }_{238} \mathrm{U}$ in the $\mathrm{UO}_{2}$ fuel regions (the $3 \mathrm{rd}$ and 7 th ring in Fig. 4 ) and the maximum difference is less than $1.8 \%$. The difference of k-infinity between NECP-X and the MC code is $-68 \mathrm{pcm}$. The results show good accuracy of NECP-X in treating the multi-annular fuel pin cell problem.

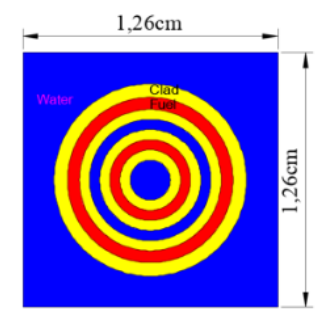

Figure 4. The geometry of the multi-fuel pin cell problem

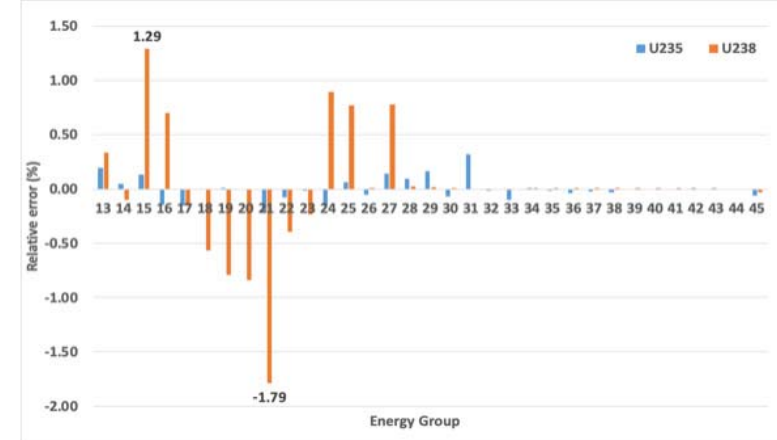

(a) The 3rd ring

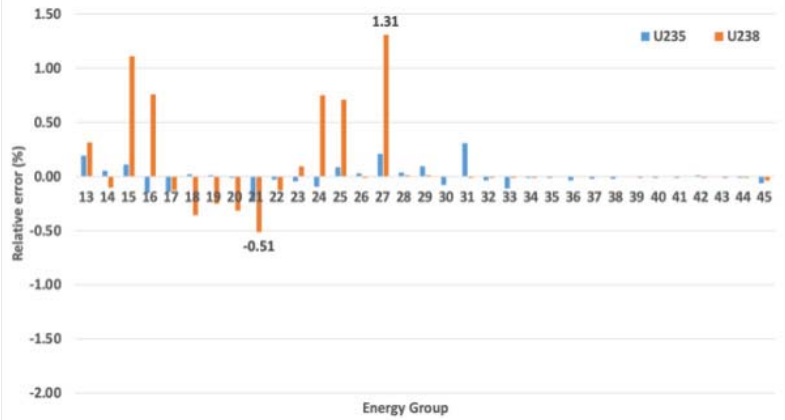

(b) The 7 th ring

Figure 5. The absorption cross sections difference of the multi-fuel pin cell problem

\subsection{A plate fuel pin cell problem}

The plate fuel problem is a $\mathrm{UO}_{2}$ fuel plate with $10.25 \mathrm{wt} \% \mathrm{U}-235$ problem. It uses aluminum as clad and light water as moderator. The temperatures and boundary condition are same as the multi-annular fuel problem. The geometry of the problem is shown in Fig. 6.

Fig. 7(a) shows the relative difference of group-wised microscopic absorption cross-section of $238 \mathrm{U}$ in the $\mathrm{UO}_{2}$ fuel region and the maximum difference is less than $1.5 \%$ which located in the 23 rd group. Fig. 7(b) shows the relative difference of group-wised microscopic absorption cross-section of $235 \mathrm{U}$ in the $\mathrm{UO}_{2}$ fuel region and the maximum difference is less than $0.66 \%$. The difference of $\mathrm{k}$-infinity between NECP-X and the MC code is $104 \mathrm{pcm}$. The results calculated by NECP-X agree well with those of the MC code. 


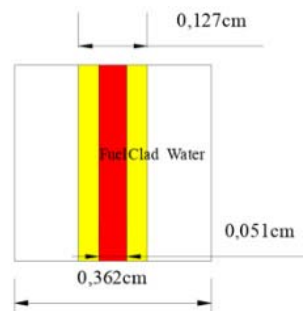

Figure 6. The geometry of one plate fuel pin cell problem

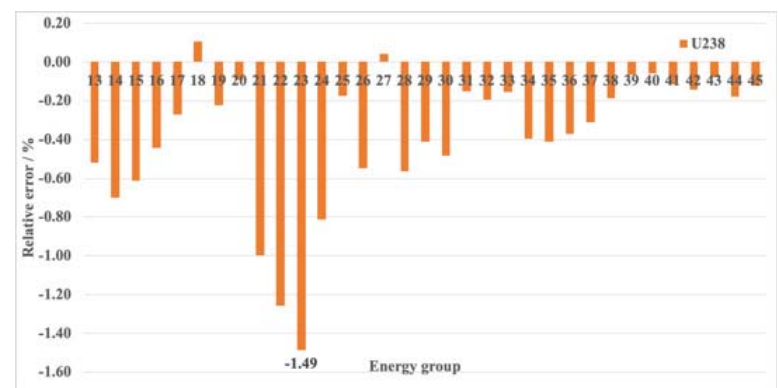

(a) $\mathrm{U} 238$

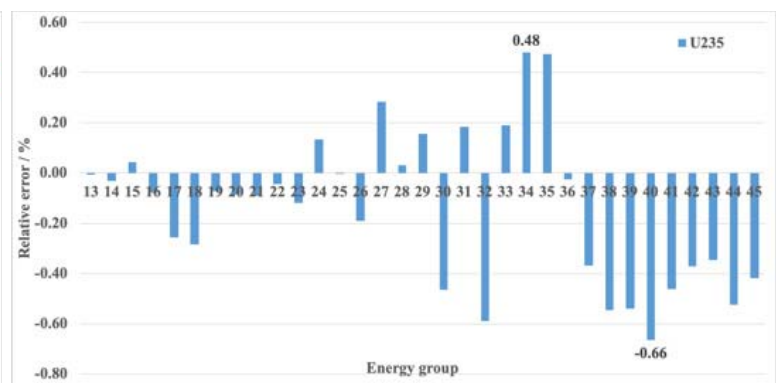

(b) U235

Figure 7. The absorption cross sections difference of one plate fuel pin cell problem

\subsection{Multi-annular fuel assembly with burnup problem}

The multi-annular assembly problem has a 17 x 17 configuration containing multi-annular fuel pin cells. The power density is $40 \mathrm{MW} / \mathrm{tU}$ and the problem is irradiated to $40 \mathrm{GWd} / \mathrm{tU}$. The $k$ eff and the difference are shown in Fig. 8. The maximum difference in $k$ eff for all depletion points is $126 \mathrm{pcm}$. The difference of power distributions at $0 \mathrm{GWd} / \mathrm{tU}, 6 \mathrm{GWd} / \mathrm{tU}$ and $40 \mathrm{GWd} / \mathrm{tU}$ are shown in Fig. 9, in which the black areas represent the guide tubes and $\mathrm{B} 4 \mathrm{C}$ rods. The maximum difference in power distribution is less than $0.41 \%$. The results indicate that the good performance of NECP-X on treating multi-annular fuel assembly with burnup problem.

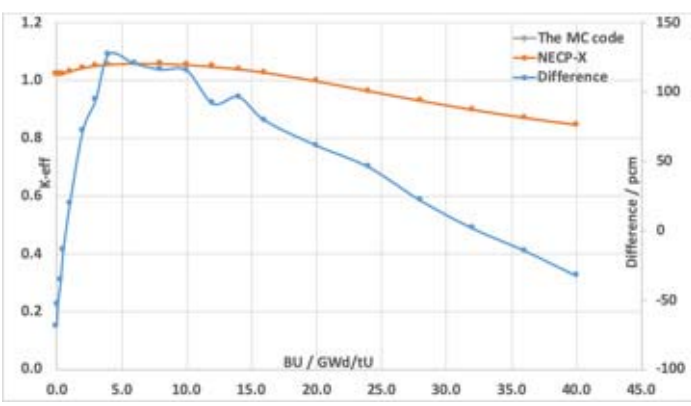

Figure 8. The $k_{\text {eff }}$ and difference for multi-annular fuel assembly with burnup problem
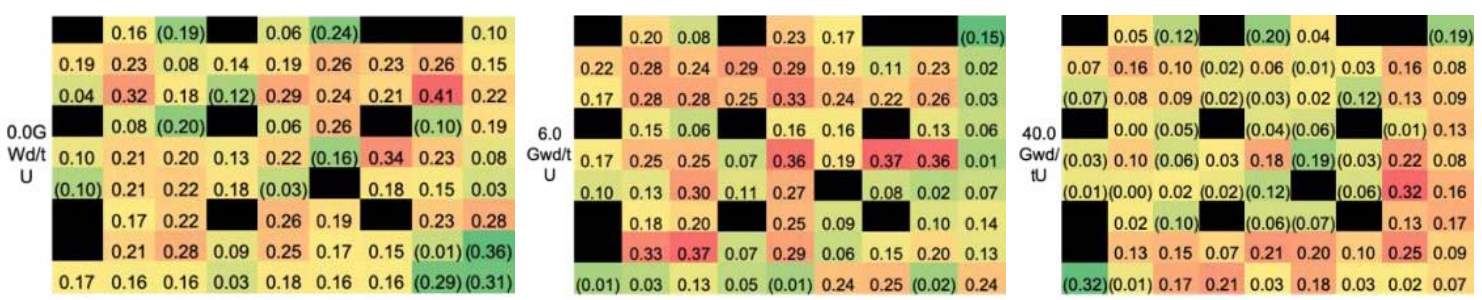

Figure 9. The pin power difference (\%) for multi-annular fuel assembly (1/4) with burnup problem 


\subsection{JRR-3M standard fuel assembly with burnup problem}

In order to further clarify the accuracy of NECP-X, the JRR-3M standard fuel assembly is tested in this work. The geometry of the standard fuel assembly is shown in Fig. 10. The $\mathrm{U}_{3} \mathrm{Si} 2$ fuel with $19.75 \mathrm{wt} \% \mathrm{U}-$ 235 is used and the final average burnup of the assembly is 550 EFPD (Effective Full Power Days). As shown in Fig. 11, the maximum difference in $k_{\text {eff }}$ for all depletion points is $201 \mathrm{pcm}$. The difference of plate power distributions and mesh power distributions at 0 EFPD, 160 EFPD and 460 EFPD for the lower right quarter assembly are provided in Fig. 12 and Fig. 13. The maximum difference in plate power distribution is less than $0.16 \%$. The maximum difference in mesh power distribution is less than $0.81 \%$. These results show that NECP-X can treat plate fuel assembly with burnup problem well.

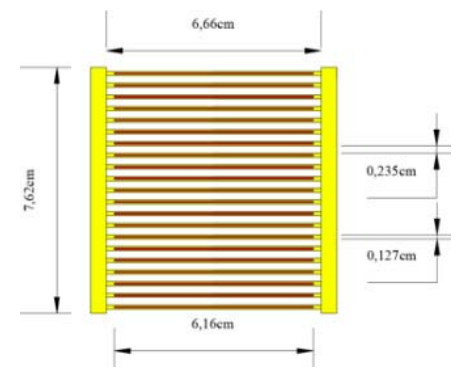

Figure 10. The geometry of JRR-3M standard fuel assembly

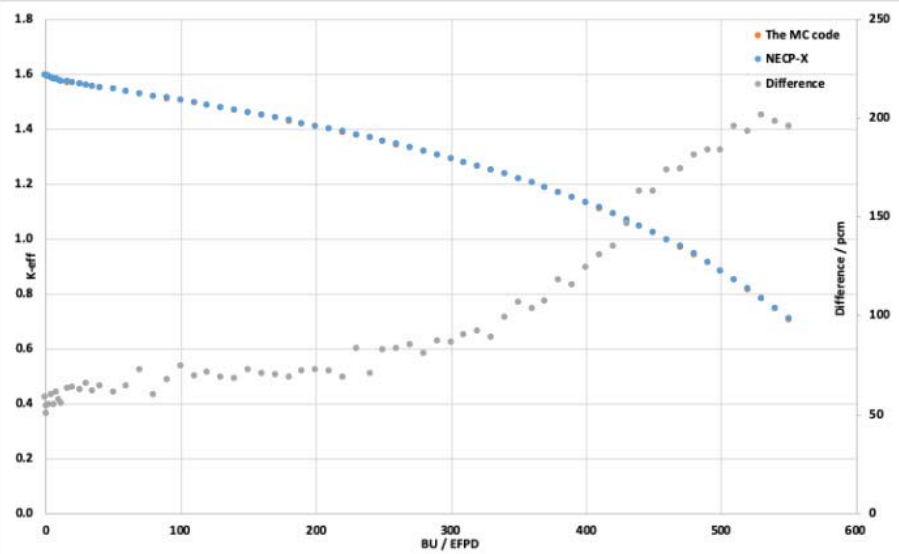

Figure 11. The $k_{\text {eff }}$ and difference for JRR-3M standard fuel assembly with burnup problem

\begin{tabular}{|c|c|c|c|c|c|c|c|c|}
\hline & 0 EFPD & & & 160 EFPD & & \multirow{2}{*}{\multicolumn{3}{|c|}{460 EFPD }} \\
\hline$=C P-X$ & The $M C$ code & Difference & NECP- & The MC code & Difference & & & \\
\hline 0.98485 & 0.98465 & $0.02 \%$ & 0.98916 & 0.98939 & $-0.02 \%$ & NECP-X & The MC code & Difference \\
\hline 0.98539 & 0.98476 & $0.06 \%$ & 0.98961 & 0.99061 & $-0.10 \%$ & 1.00658 & 1.00606 & $0.05 \%$ \\
\hline 0.98650 & 0.98612 & $0.04 \%$ & 0.99053 & 0.99089 & $-0.04 \%$ & 1.00632 & 100643 & $-0.01 \%$ \\
\hline 0.98829 & 0.98918 & $-0.09 \%$ & 0.99199 & 0.99208 & $-0.01 \%$ & 1.00572 & 1.00574 & $0.00 \%$ \\
\hline 0.99093 & 0.99140 & $-0.05 \%$ & 0.99405 & 0.99423 & $-0.02 \%$ & 1.00482 & 1.00529 & $-0.05 \%$ \\
\hline 0.99467 & 0.99459 & $0.01 \%$ & 99686 & 0.99727 & $-0.04 \%$ & 1.00341 & 1.00321 & $0.02 \%$ \\
\hline .00000 & 0.99964 & $0.04 \%$ & 1.00066 & 1.00073 & $-0.01 \%$ & 1.00139 & 1.00075 & $0.06 \%$ \\
\hline 00777 & 1.00851 & $-0.07 \%$ & 1.00 & 1.00517 & $0.06 \%$ & 0.99865 & 0.99930 & $-0.06 \%$ \\
\hline 01975 & 1.02024 & $-0.05 \%$ & 08 & 1.01307 & $0.00 \%$ & $\begin{array}{l}.99461 \\
.98853\end{array}$ & 0.99493 & $-0.03 \%$ \\
\hline 4185 & 1.04092 & $0.09 \%$ & 1.02826 & 1.02656 & $0.16 \mathrm{~b}$ & 0.98998 & 0.98937 & $0.06 \%$ \\
\hline
\end{tabular}

Figure 12. The plate power and its difference for JRR-3M standard fuel assembly (1/4) with burnup problem 


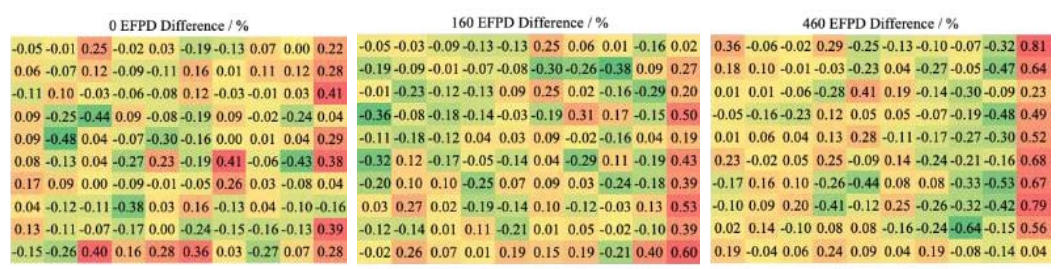

Figure 13. The mesh power and its difference for JRR-3M standard fuel assembly (1/4) with burnup problem

\section{CONCLUSIONS}

In order to deal with complex geometric fuels with the Global-Local method used in NECP-X, the new improved Global-Local method is proposed and verified in this paper. Based on the newly implemented CSG (Constructive Solid Geometry) method, the complex geometry can be explicitly modeled. Moreover, the equivalent one-dimensional plate or one-dimensional cylindrical problem for each pin cell is constructed for the local problems. The pseudo-resonant-nuclide subgroup method is utilized as the local solver to generate the effective shielding cross sections. The multi-annular fuel problems and plate fuel problems are tested. The numerical results show good consistence of NECP-X and the Monte Carlo code in predicting microscopic cross sections, the eigenvalue and the power distributions.

\section{ACKNOWLEDGMENTS}

This work is supported by the National Natural Science Foundation of China (No. 11605130 and 11735011).

\section{REFERENCES}

1. Jun Chen, et al, "A new high-fidelity neutronics code NECP-X," Annals of Nuclear Energy, 116, pp. 417-428 (2018).

2. Zhouyu Liu, Qingming He, et al, "The Pseudo-Resonant-Nuclide Subgroup Method Based GlobalLocal Self-Shielding Calculation Scheme," Journal of Nuclear Science and Technology, 55:2, pp. 217228 (2018).

3. Zhouyu Liu, Chen Zhao, et al. "The material-region-based 2D/1D transport method," Annals of Nuclear Energy, 128, pp. 1-11 (2019).

4. Zhouyu Liu, et al, "A new three-dimensional method of characteristics for the neutron transport calculation," Annals of Nuclear Energy, 38, pp. 447-454 (2011).

5. Youqi Zheng, et al, "A new approach to three- dimensional neutron transport solution based on the method of characteristics and linear axial approximation," Journal of Computational Physics, 350, pp. 25-34 (2017).

6. Lu Cao, et al, "One Step Whole Core Calculation of 3D Complex Geometrical Small PWR Based on NECP-X," Nuclear Power Engineering, 39, pp. $92-97$ (2018).

7. Xinyu Zhou, et al, "Method Based On Characteristic Ray Information FOR Arbitrary Geometry Volume Calculation in Necp-x," Journal of Nuclear Engineering and Radiation, on line (2019).

8. Sugimura N, Yamamoto A, "Evaluation of Dancoff factors in complicated geometry using the Method of Characteristics," Journal of Nuclear Science and Technology, 43, pp. 1182-1187 (2006).

9. Qian Zhang, et al, "An Improved Resonance Self- Shielding Calculation Method Based on Equivalence Theory," Nuclear Science and Engineering, 173, pp. 233-252 (2015).

10. Hyung Jin Shim, et al, "McCARD for neutronics design and analysis of research reactor cores," Annals of Nuclear Energy, 82, pp. 48-53 (2015).

11. Askew J R, et al, "A general description of lattice code WIMS," J. Brit. Nucl. Energy Soc. 5, pp. 546585 (1966). 